This article has been scanned by iThenticat No plagiarism detected

Volume 3, Issue 4, August 2021

p. 194-205

\title{
THE EFFECT OF DIGITAL EDUCATIONAL GAMES ON THE MOTIVATION TOWARDS E-LEARNING AND LIFE SKILLS OF PRIMARY SCHOOL STUDENTS IN ARAB SOCIETIES
}

http://dx.doi.org/10.47832/2757-5403.4-3.17

\section{Kareema Abd Al Kareem ALI ${ }^{1}$ \& Amal Zuheir SAWALHA ${ }^{2}$}

\begin{abstract}
:
This study investigates the effect of employing digital educational games (DEGs) on motivation towards e-learning, and life skills of primary school students in more than one Arab context: in Palestine, Jordan, Bahrain, and United Arab Emirates. It also investigates the need to employ these games in the educational process, the challenges which faced the employment of these games, and ways to develop their employment in the learning process. . This qualitative study used a multiple case study by conducting open, semi-structured interviews on a sample of four teachers and four parents of two from each country to reveal in-depth views to clarify the mechanism of employing these digital educational games. Data were subjected to multiple case study analysis using Ary et al. (2010) model. The results show that DEGs were a contributing factor in increasing students' motivation towards elearning and enabling them to acquire the life skills necessary to live in this era when adding these games to the elements of fun and excitement to learn in a collaborative environment. They provide students with communication, problem-solving, decision-making and technological skills. The results also show the challenges that face employing them in the e-learning process, represented in material ones, such as the weakness of the technological infrastructure, and educational ones, such as the weakness of teachers in dealing with DEGs and choosing the appropriate game for the age category and subject. The study recommends adopting the employment of DEGs officially in public education institutions, and providing material and technical support to teachers, students and parents.
\end{abstract}

\footnotetext{
${ }^{1}$ Researcher, An-Najah National University, Palestine, kareemanasir78@gmail.com, https://orcid.org/00000003-3645-1949

${ }^{2}$ Researcher, An-Najah National University, Palestine, amalarom2013@gmail.com, https://orcid.org/0000$\underline{0001-8413-955 X}$
}

Copyright (C) Published by IJHER Journal, www.ijherjournal.com Rimar Academy, Fatih, Istanbul, 34093 Turkey

All rights reserved 
Key words: Digital Educational Games, E-Learning, Motivation Towards Learning, Life Skills, Arab Society.

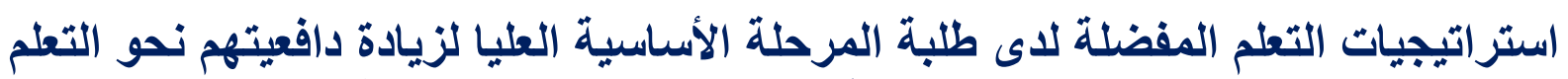

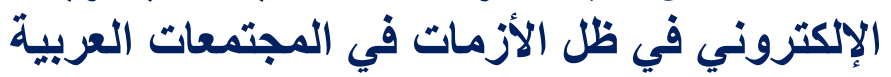

\author{
كريمة علي 3

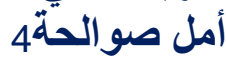

تناقش هذه الدر اسة استر اتيجيات التعلم المفضلة لدى طلبة المرحلة المبلة الأساسية العليا وتأثير ها

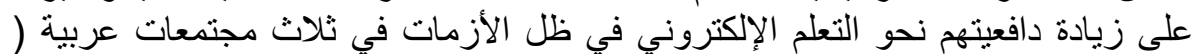

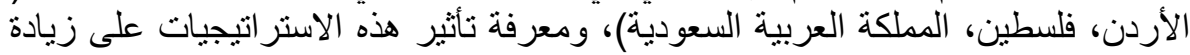

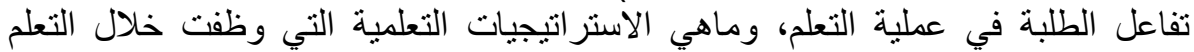

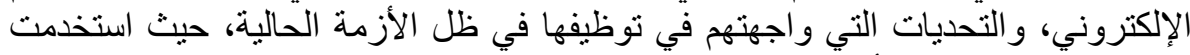

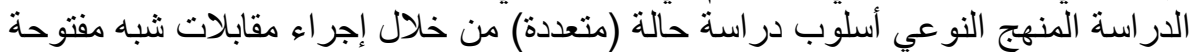

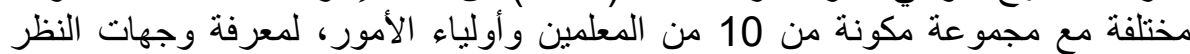

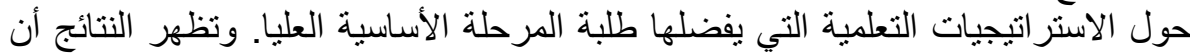

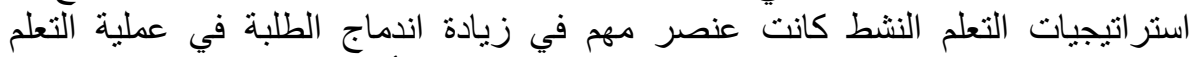

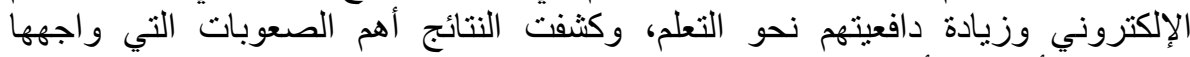

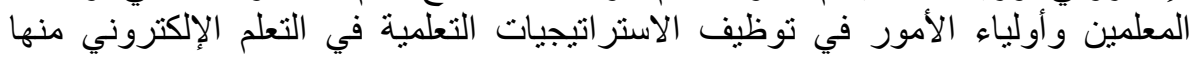

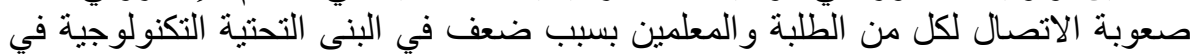

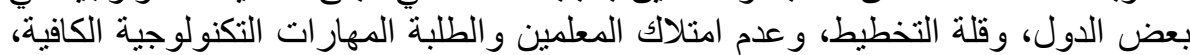

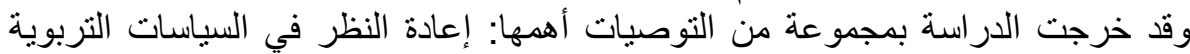

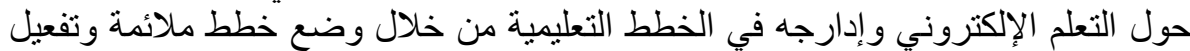

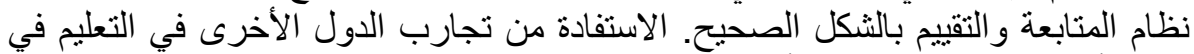

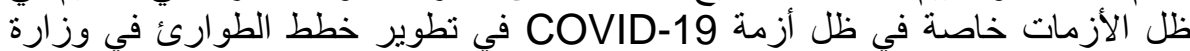

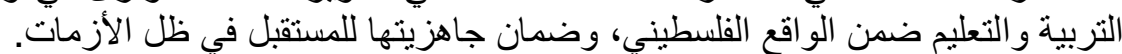

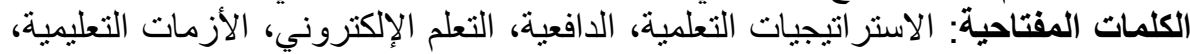

المجتمعات العربية..

المقدَّمة:

تميز هذا العصر بالنمو التكنولوجي والعلمي المتسارع الذي أدى إلى انفجار ثورة علمية كبيرة في المجالات التربوية

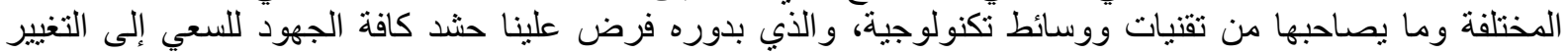

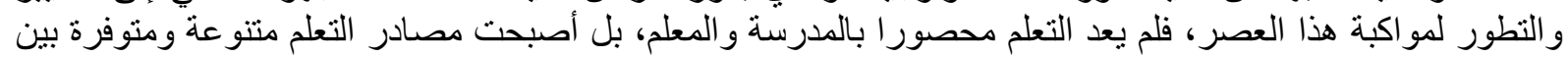

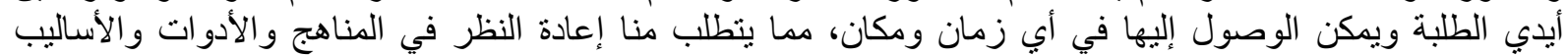

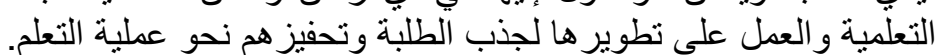

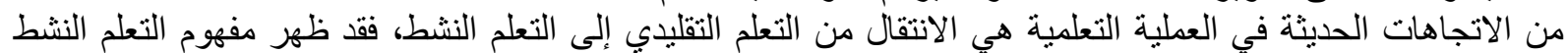

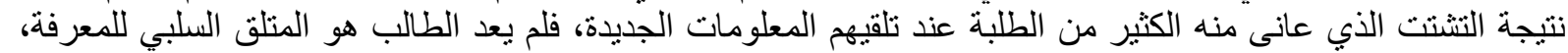

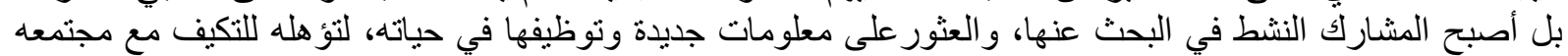

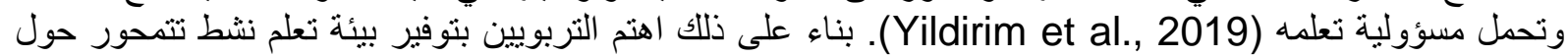

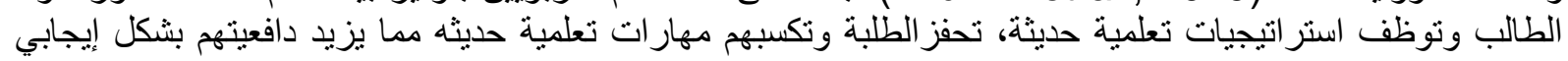

نحو عملية التعلم (University, 2020). 


\section{THE EFFECT OF DIGITAL EDUCATIONAL GAMES ON THE MOTIVATION \\ TOWARDS E-LEARNING AND LIFE SKILLS OF PRIMARY SCHOOL STUDENTS \\ IN ARAB SOCIETIES}

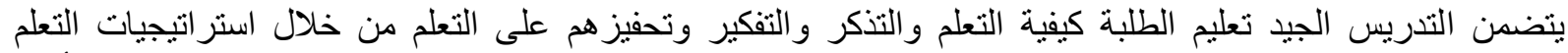

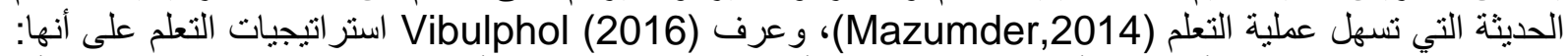

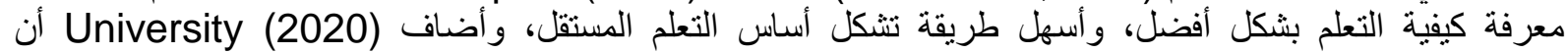
استراتيجيات التعلم مجموعة من التكتيكات العقلية التي يستخدمها المعلمون لتوصيل المعرفة والمهارات للطلبة بشكل

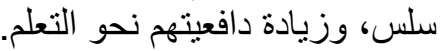

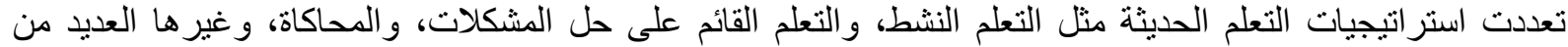
الاستراتيجيات والتي اعتمدت في هدفها على النظرية البنائية، بحيث يتحمل المتعلم فيها مسؤولية تنظيم عملية التئلية التعلم

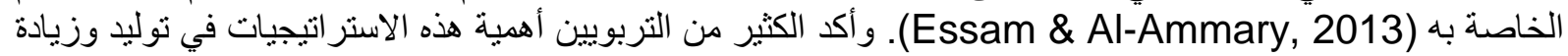

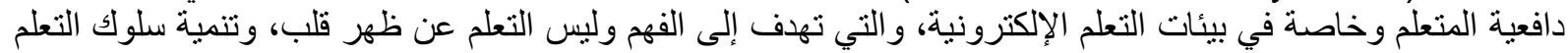

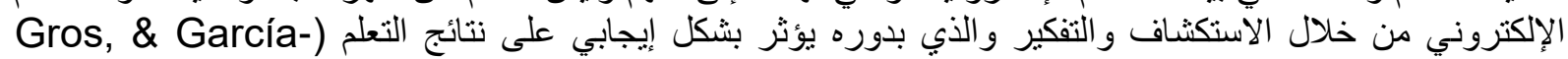

(Peñalvo, 2016

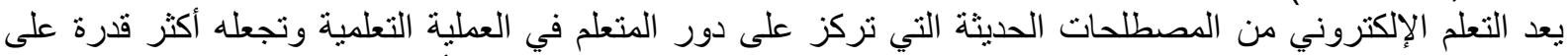

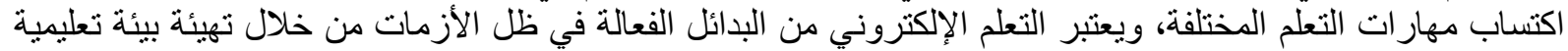

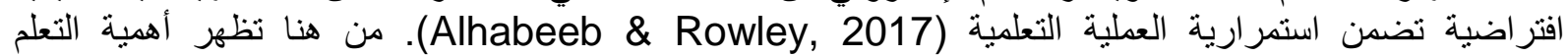
الإلكتروني كضرورة تفرضها الأزمات على اختلافها كجائحة كورونا ولما يتمتع به من خصائه التهائص تجعله أكثر ملائمة

لتجنب أضرار هذه الجائحة على العملية التعلمية (Murphy,2020).

مشكلة الدراسة الازية

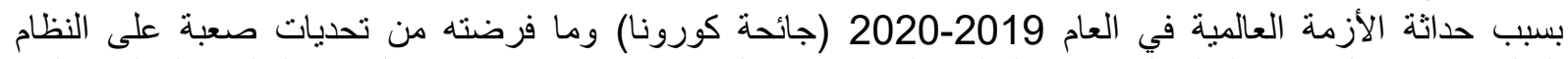

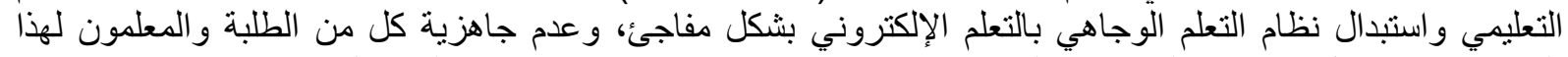

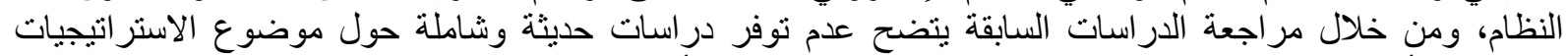

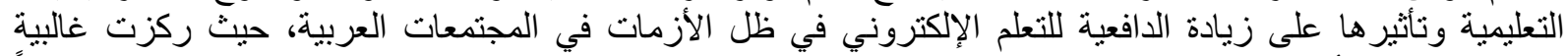

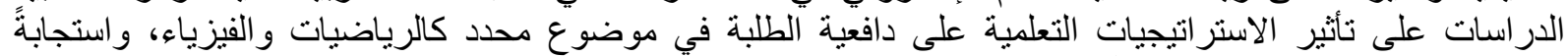

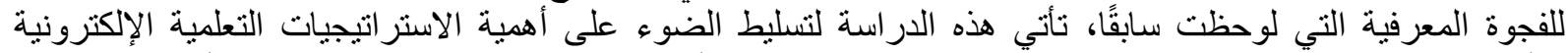

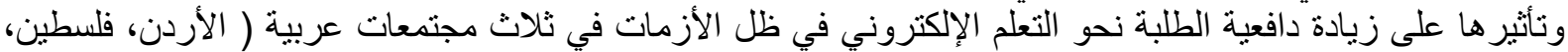

المملكة العربية السعودية).

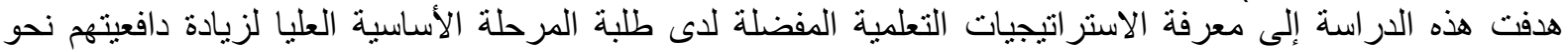

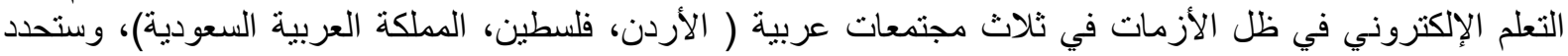
الدراسة كيف وظفت هذه المجتمعات الاستر اتيجيات التعلمية في التعلم الإلكتروني للعمل على الإنى زيادة دافعية طلبة المرحلة

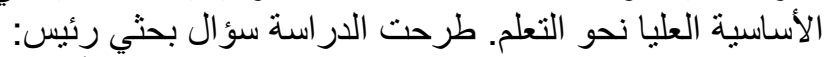

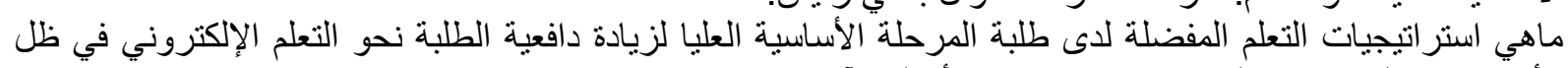

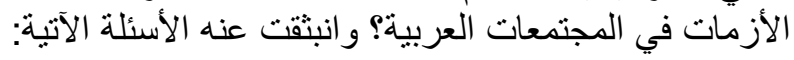

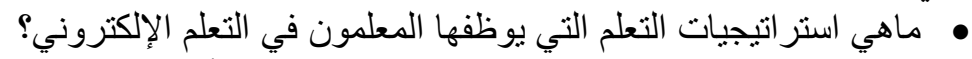

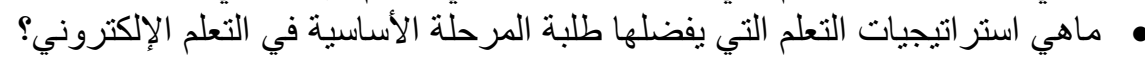

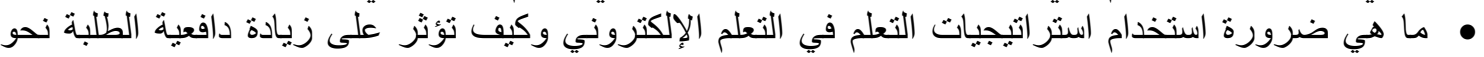

التعلم?

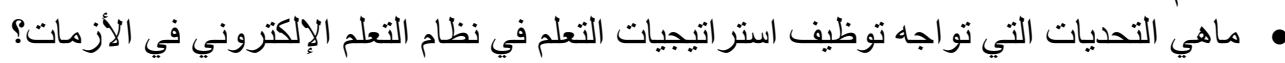

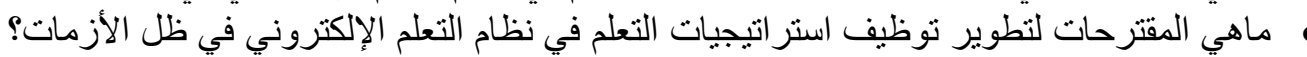

\footnotetext{
الأدب النظري

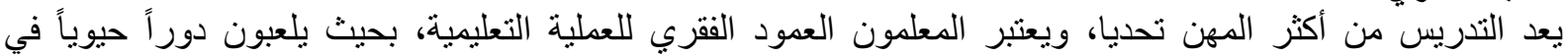

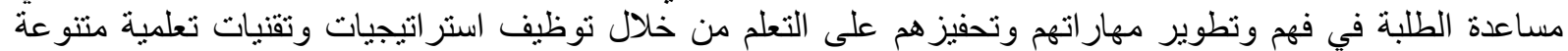

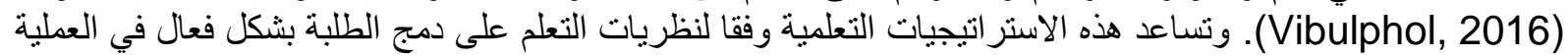

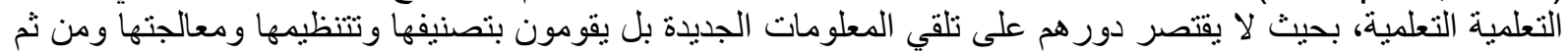


إدراكها باستخدام العمليات العقلية لديهم (Shi, 2017). وتتميز الاستراتيجيات التعلمية أنها نراعي الفروق الفردية،

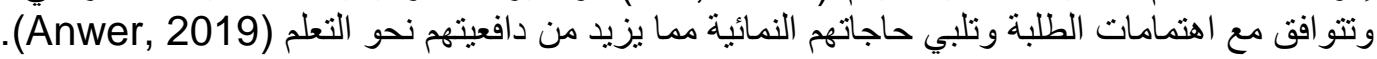

استراتيجيات التعلم وأهميتها.

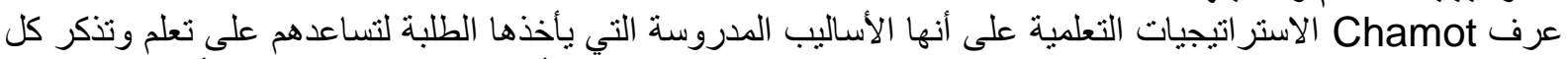

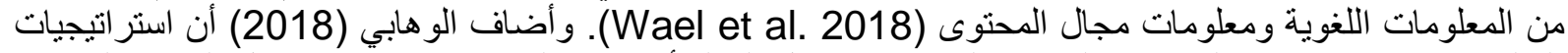

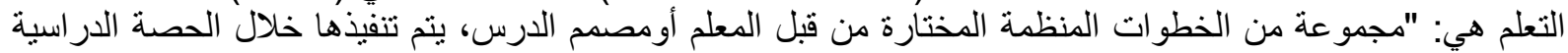

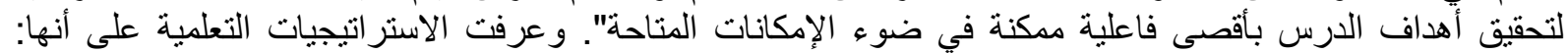

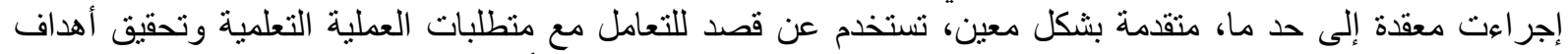

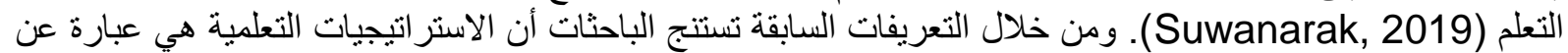

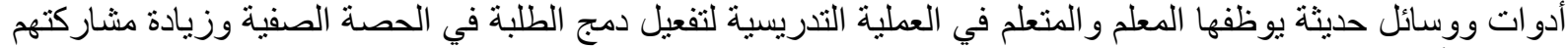
لتحقيق الأهداف التربوية المنشودة. استراتيجيات التعلم ليست تقنيات تهدف إلى مساعدة الطلبة في تعلمهم فقط، بل يمكن اعتبار ها مناهج لتعلم السلوك

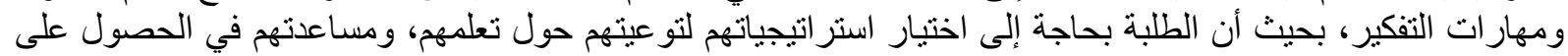

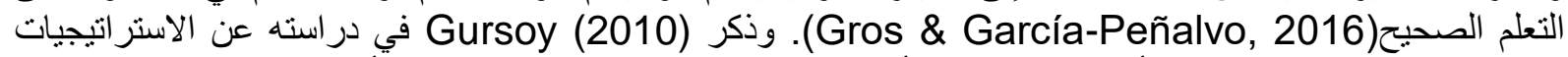

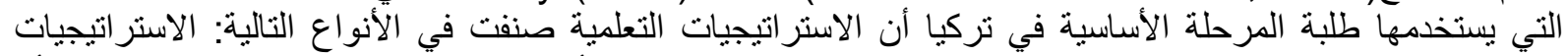

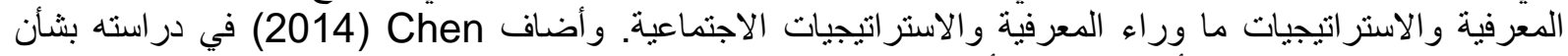

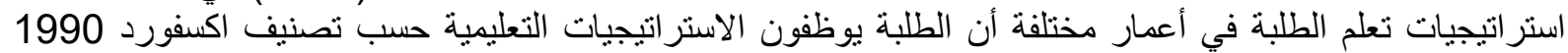

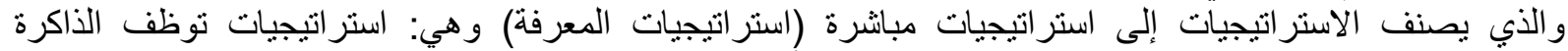

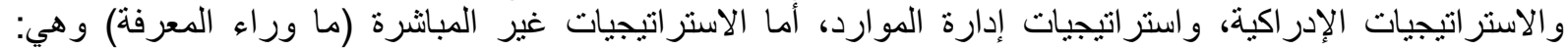

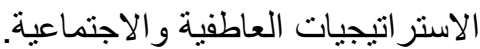

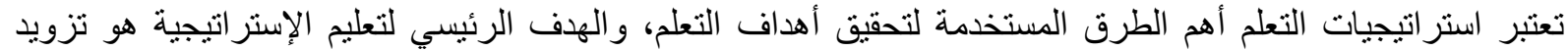

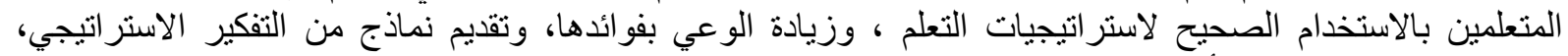

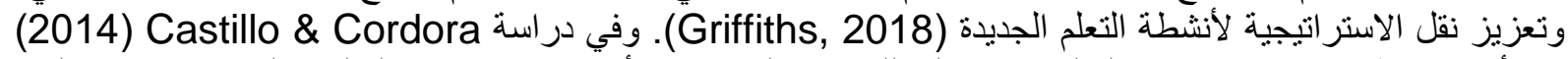

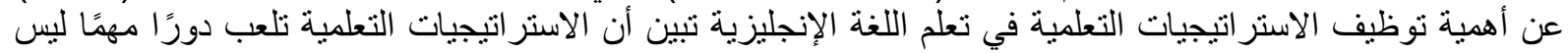

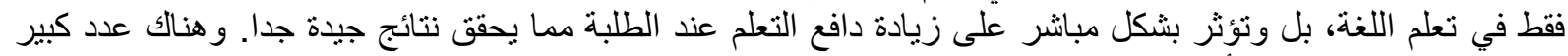

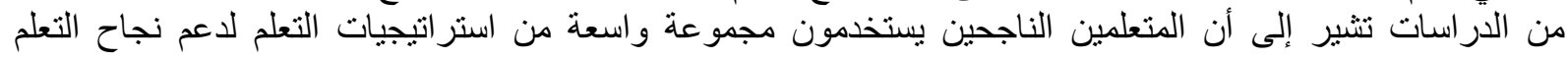

كدر اسة (Dörnyei \& Ryan, 2015).

الا(فعية

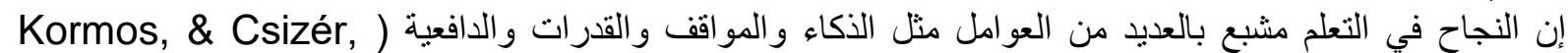

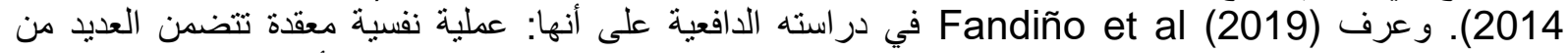

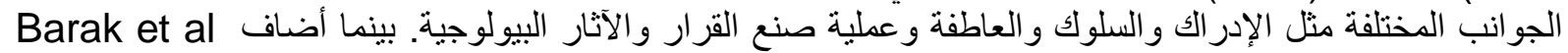

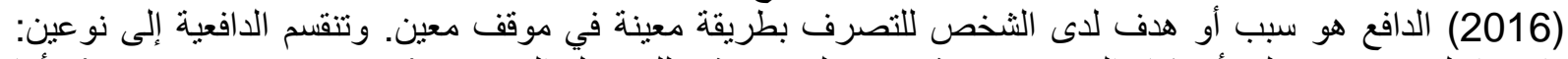

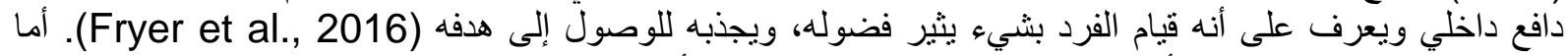

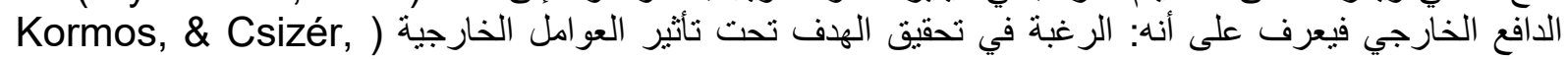

(2014 تظهر الكثبر من الأدبيات النظرية كما في دراسة (2016) Barker et al أوضح أن الطلبة ذوب الدافعية المرتفعة في

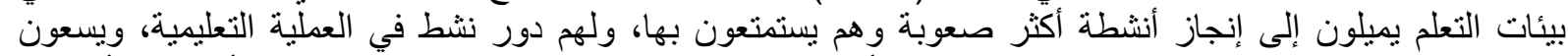

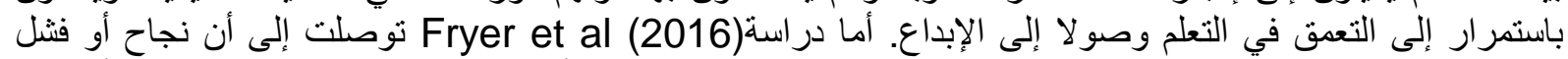

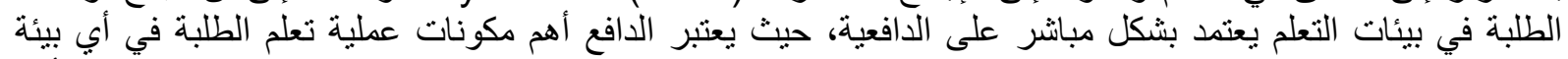

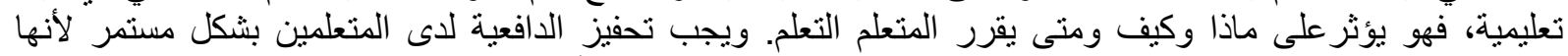

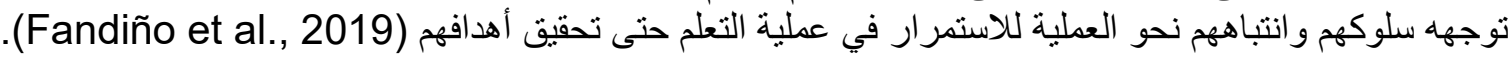

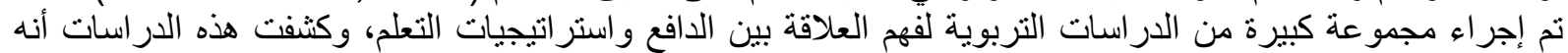

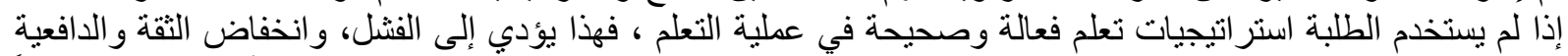

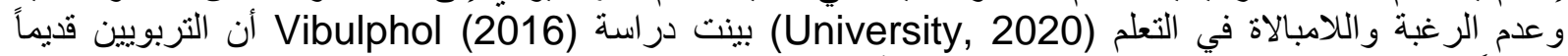

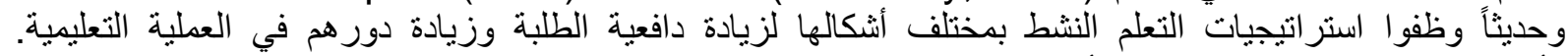

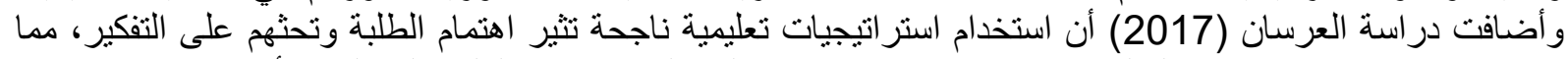

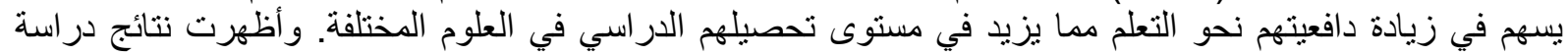


Saraçoglu (2020)

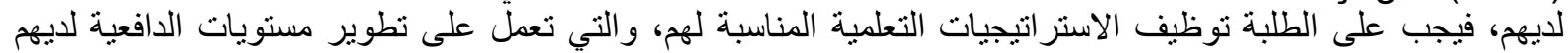
مما يعزز نتائج التحصيل لديهم.

$$
\text { التعلم الإكتروني }
$$

يتطلب ظهور التُعلم الإلكتروني استراتيجيات و أساليب جديدة للتعليم و التعلم تكون أكثر تركيزًا على المتعلم، مما يؤدي التئي

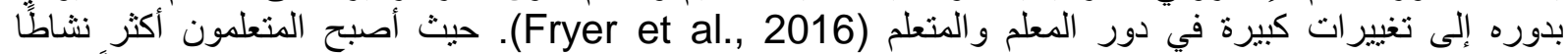

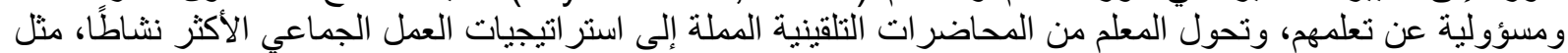

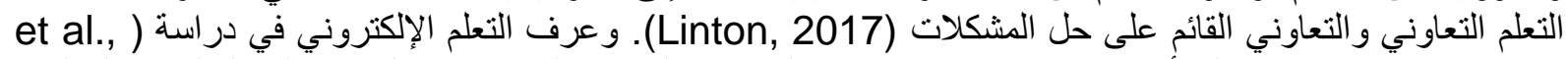

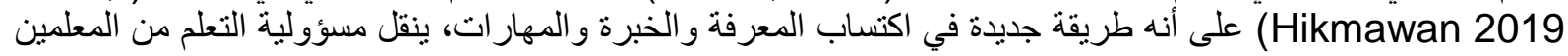

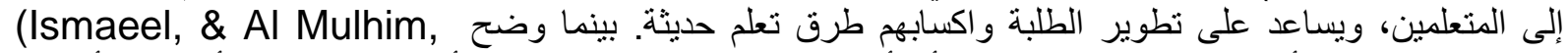

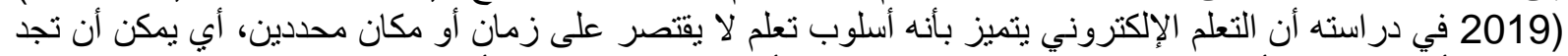

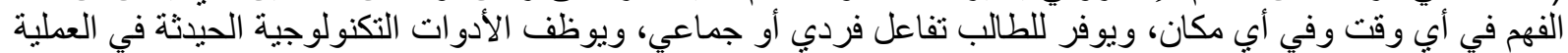
التعليمية لتحقيق أهدافها. يكون الدافع في بيئات التعلم الإلكتروني أمرًا بالغ الأهمية، وتعد خبرة الطلبة مع الأجهزة الألكية الألية، بالإضافة إلى دعم المعلم

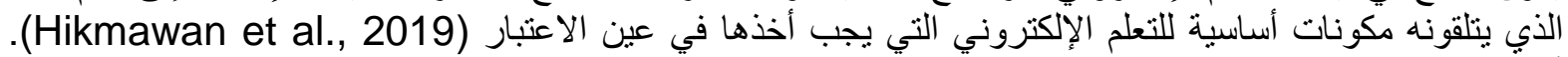

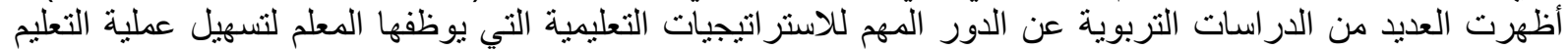
عبر الانترنت، ودعم فعالية الأدوات التكنولوجية لتسهيل فهم المحتوى التعليمي، وتعزيز التورئ المشاركة في عملية التعلم

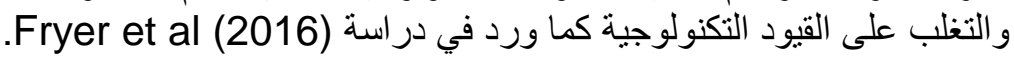

التحديات التي تواجه التعلم الإلكتروني

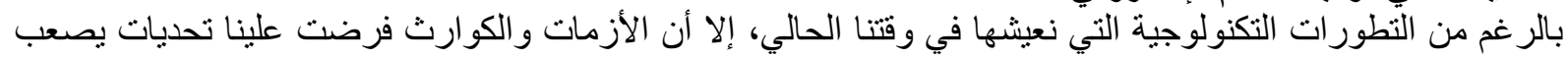

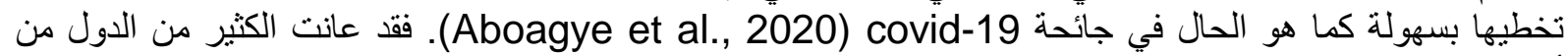
أزمات تعليمية حقيقية في ظل جائحة covid-19 نظر ال للانتشار السريع لهذا الوباء الوباء على مستوى العالم، وعدم جاهزية

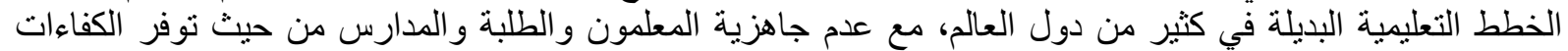

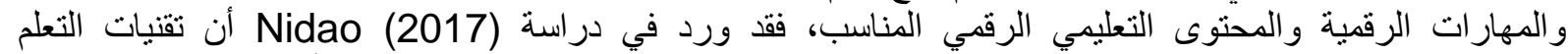

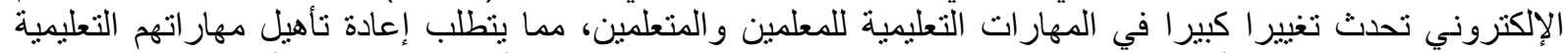

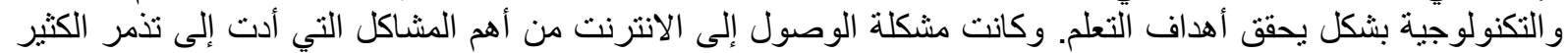

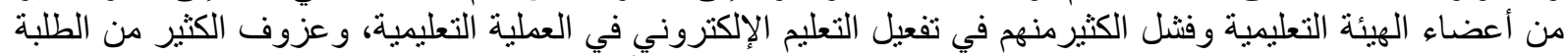

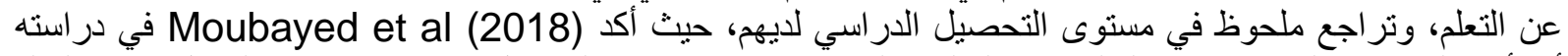

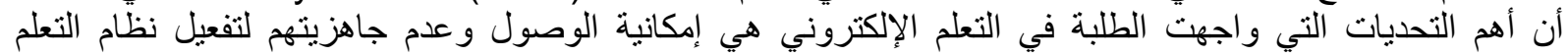

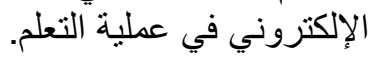

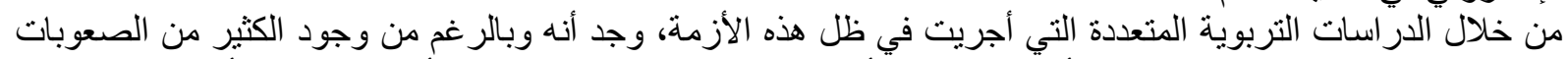

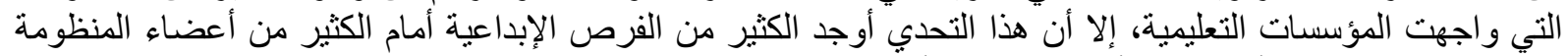

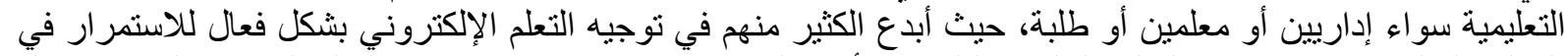

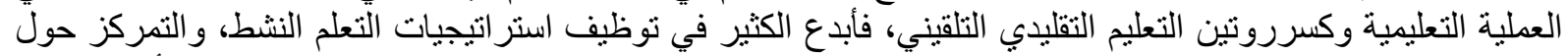

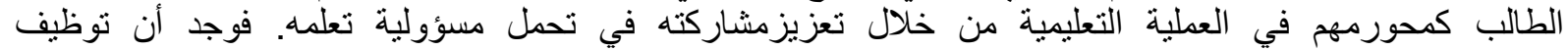

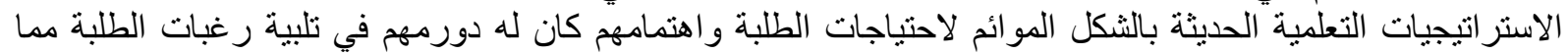

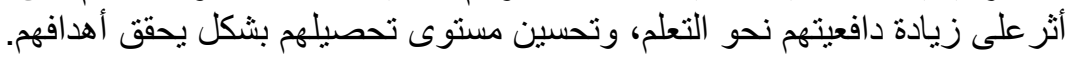

هنالك عدد من الدّر اسات التجريبية كدر اسة (2012) Hikmawan et al وجدت أن التبات العديد من ممارسات التعليمية في

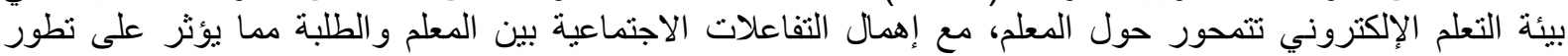

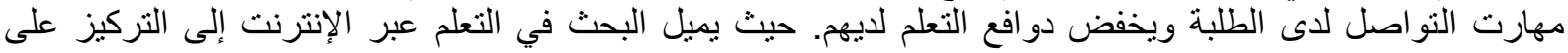

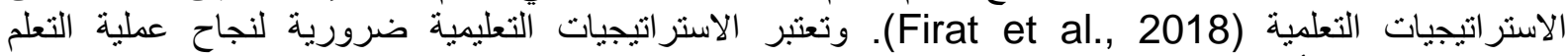
الإلكتروني وتحقيق أهدافه (Lin et al,. 2017). ويتطلب التعلم الإلكتروني من الطلبة ضبط سلوكهم التعلمي، وتحديد 
استر اتيجياتهم التعلمية، وتحديد أهدافهم لزيادة تحفيزهم نحو التعلم (Alhabeeb \& Rowley, 2017). ومن منظور

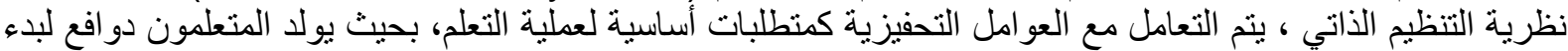

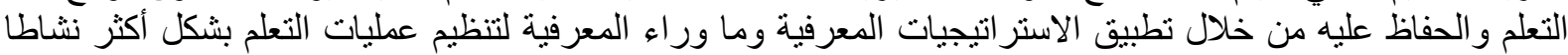

.(Zimmerman, 2012) وبالتالي فإن الإطار المفاهيمي الذي تقوم عليه هذه الدراسة يعتبر توظيف مجموعة متنوعة من استراتيجيات التعلم

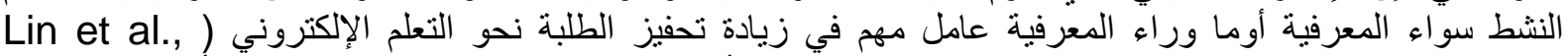

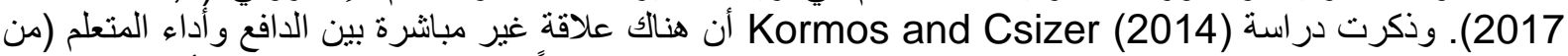
خلال استخدام الاستر اتيجيات المعرفية) ، وتلعب استر اتيجيات التعلم دورًا وسيطًا في العلاقة بين التحفيز وأداء أنئ المتعلم.

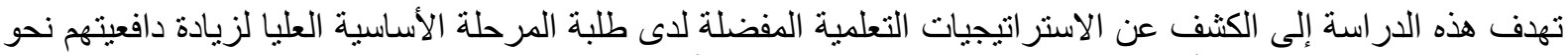

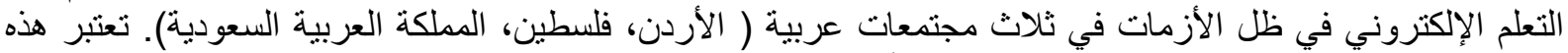

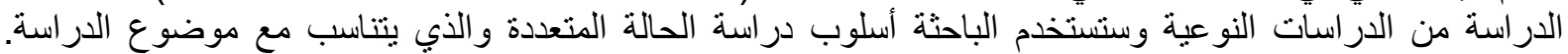

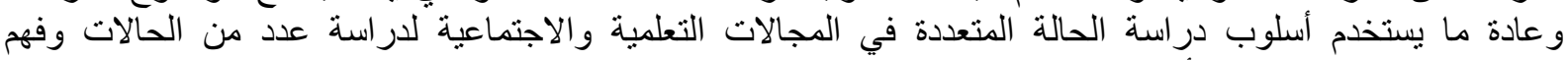

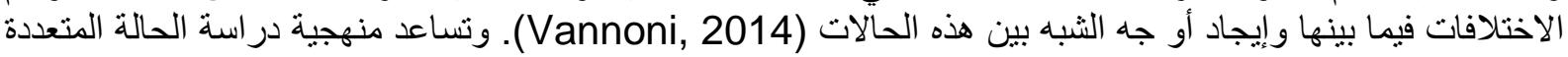

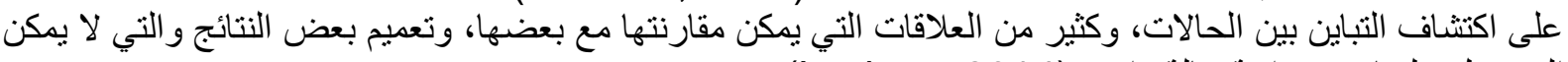
الحصول عليها من در اسة حالة واحدة (Levings, 2014).

عينة الدراسة: تكونت عينة الدراسة من اسنة 3 من أولياء الأمور، و3 معلمات في ثلاث مجتمعات عربية مختلفة ( الأردن، فلسطين، المملكة

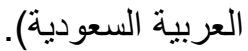
جدول (1): وصف لعينة الدراسة العية

\begin{tabular}{|c|c|c|c|c|c|}
\hline التخصص & المستوى التعليمي & المـنة & البلد & العمر & الاسم \\
\hline لغة عربية & بكالوريوس & معلمة & السعودية & 45 & سهام \\
\hline لغة انجليزية & بكالوريوس & معلمة & الأردن & 42 & رنا \\
\hline أحياء & ماجستير & معلمة & فلسطين & 43 & هبة \\
\hline هندسة & بكالوريوس & ربة منزل & السعودية & 40 & هدى \\
\hline محاسبة & دبلوم & ربة منزل & الأردن & 39 & نور \\
\hline مناهج & ماجستير & معلمة & فلسطين & 40 & هناء \\
\hline ادارة أعمال & بكالوريوس & معلم & فلسطين & 32 & محمود \\
\hline هندسة ميكانيك & ماجستير & مهندس & السعودية & 44 & إبر اهيم \\
\hline فنون تطبيقية & دكتور اة & مدرس جامعي & الأردن & 40 & مازن \\
\hline إدارة أعمال & ماجستير & أعمال حرة & فلسطين & 36 & مجدي \\
\hline
\end{tabular}

إجراءات المقابلة

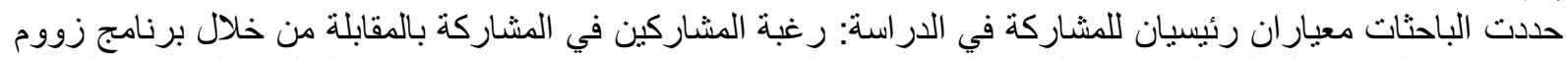

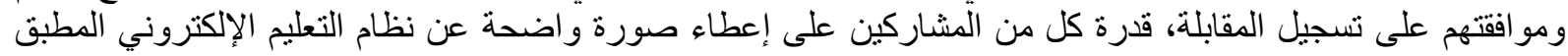

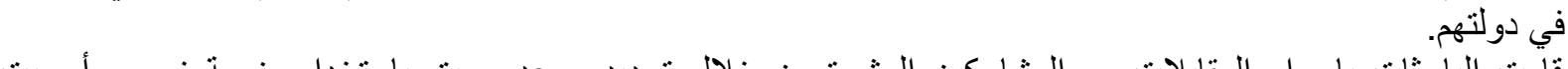

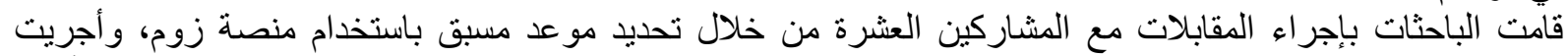

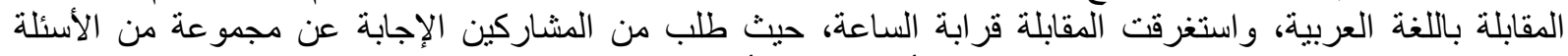

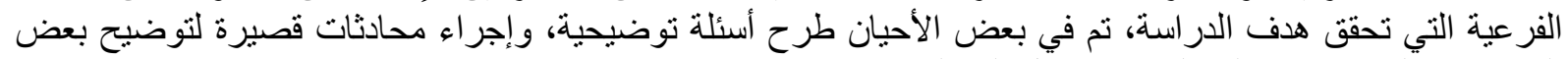
النقاط غير ألمفهومة في المقابلة، تم تسجيل المقابلة وتدوينها.

تحليل البيانات

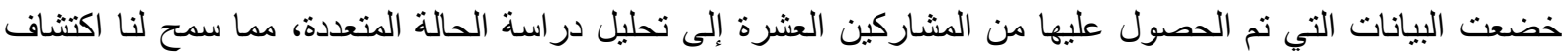

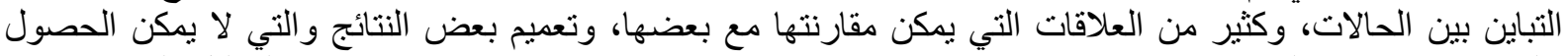

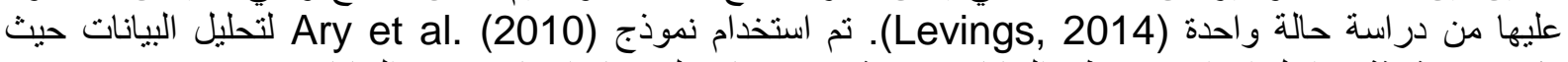
يتكون من ثلاثة مر احل ابتداءً من تتظيم البيانات ومن ثم ترميز ها وتقليصها وانتهاءً بتفسير البيانات. 
اعتمدت الدر اسة في التحليل على ثلاثة محاور رئيسية: أهية الاستر اتيجيات التعلمية في التعلم الإلكتروني، التحديات التي التي التئي

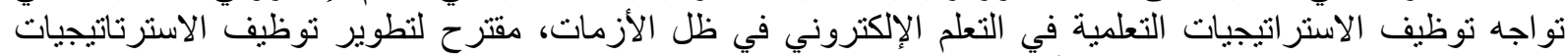
التعلمية في التعلم الإلكتروني في ظل الأنسي الأزمات.

\section{الصلاحية والموثوقية}

للتحقق من صدق النتائج، نم جمع وتحليل البيانات من قبل الباحثات وفق أسس المنهج النوعي مع مر اعاة الموضوعية

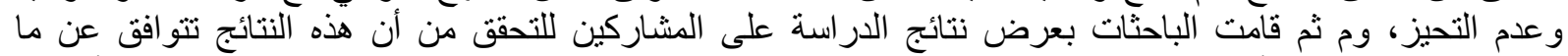

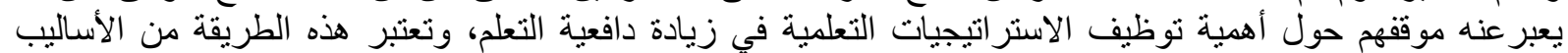

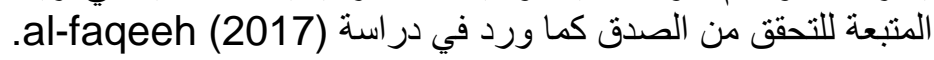

محددات الاراسة

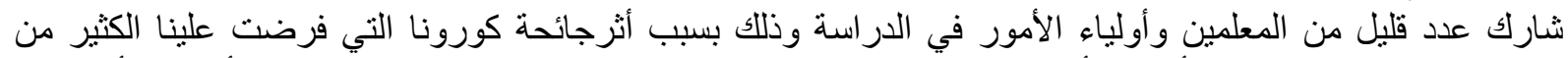

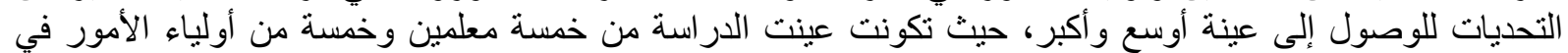

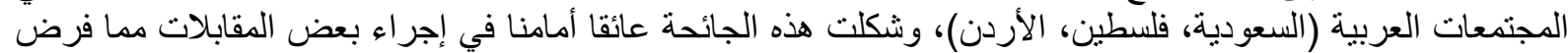

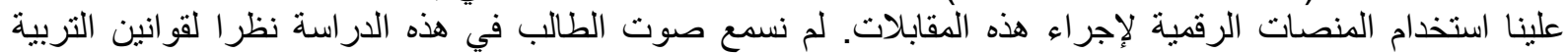

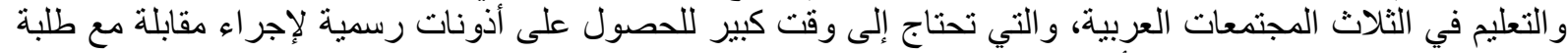
المدارس، علاوة على موافقة ولي أمر الطالب.

مناقثة النتائج

بعد تكوين الفئات الرئيسية و إيجاد العلاقات و الروابط بين هذه الفئات تلخصت نتائج الدر اسة في ثلاثة محاور رئيسية: 1 1 أهمية الاستر اتيجيات التعلية في التئية التعلم الإلكتروني.

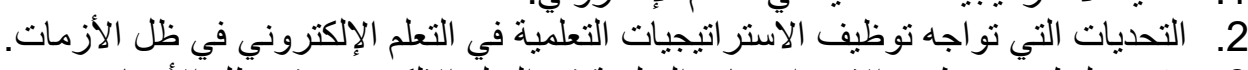

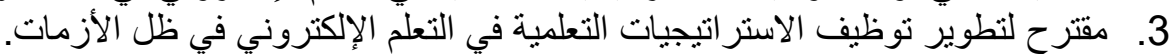

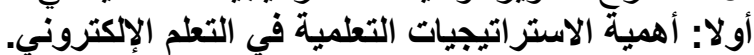

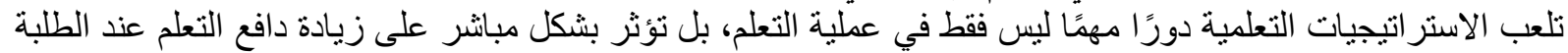

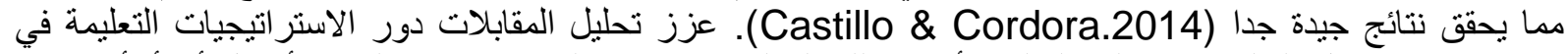

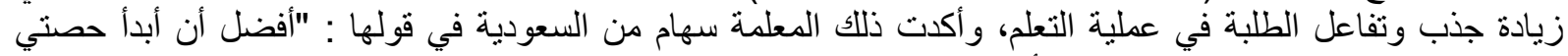

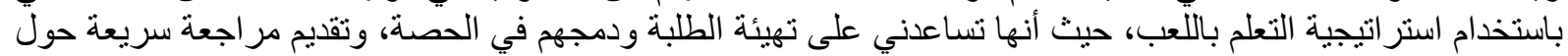

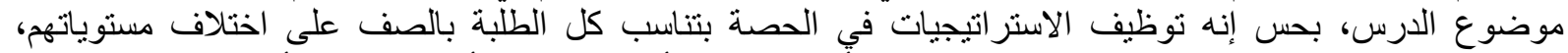

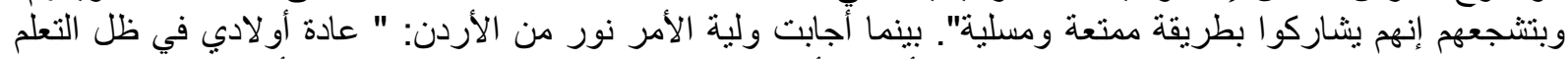

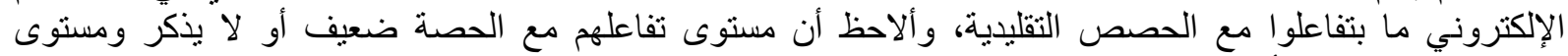

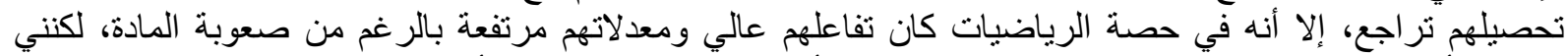

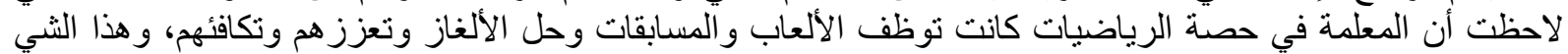

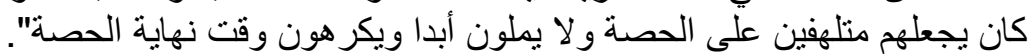

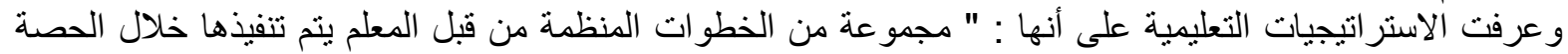

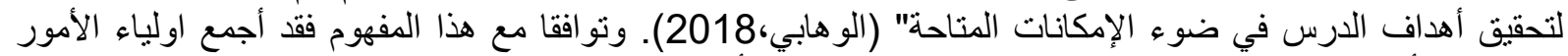

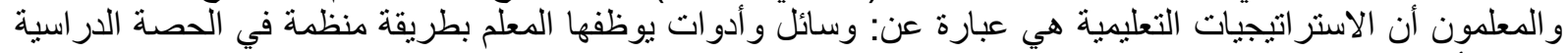

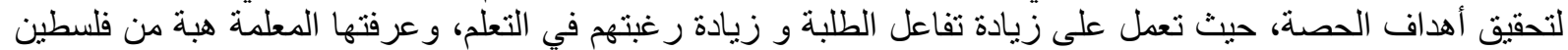

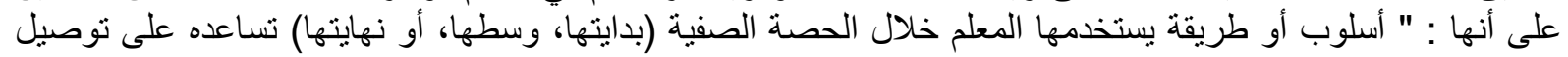

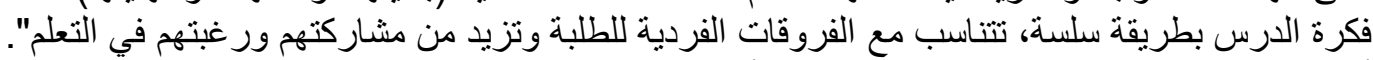

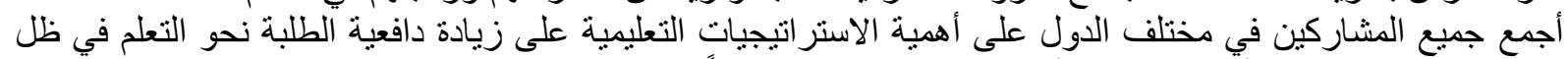

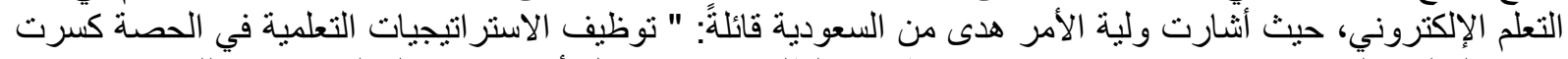

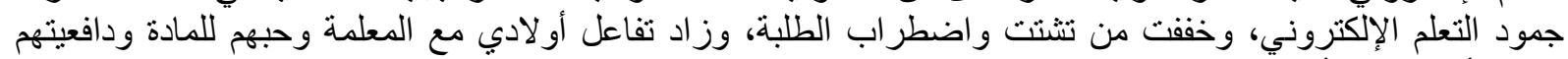

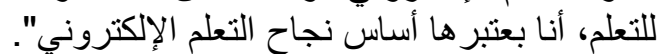
ثانيا: التحديات التي تواجها توظيف الاستراتيجيات التعلمية في التعلم الإكتروني في ظل الأزمات. 
تلخصت التحديات التي تواجه نوظيف الاستراتيجيات التعلمية في التعليم الإلكتروني في ظل فل الأزمات في ثلاثة نقاط

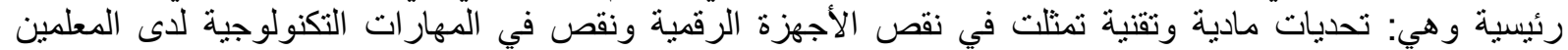

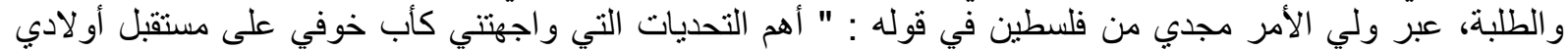

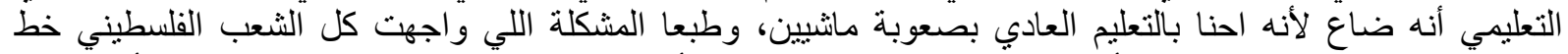

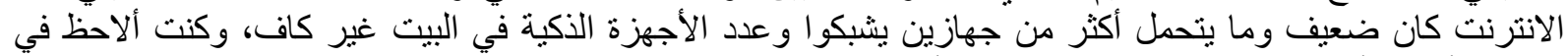

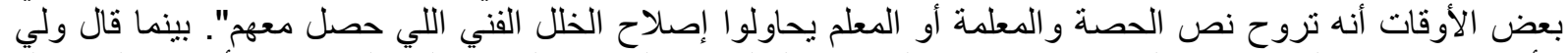

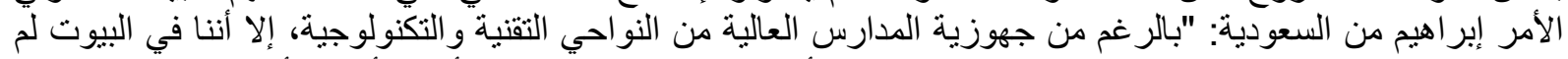

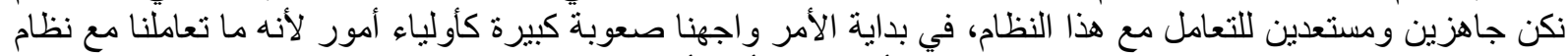

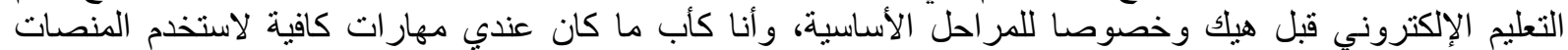

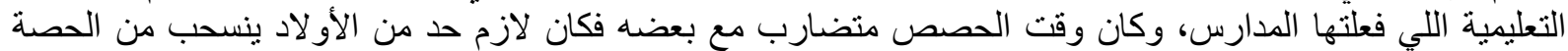

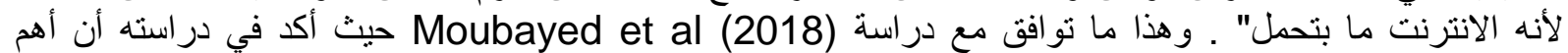
التحديات التي واجهت الطلبة في التعلم الإلكتروني هي إمكانية الوصول و عدم جاهزيتهم لتفعيل نظام التعلم الإكتروني في عملية التعلم. تمثلت تحديات التعلم الإلكتروني في تحديات نفسية أيضا واجهها أولياء الأمور والطلبة والمعلمين نتيجة لحداثة التجربة

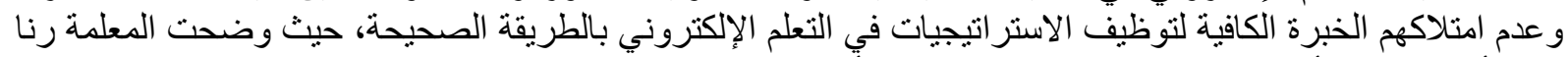

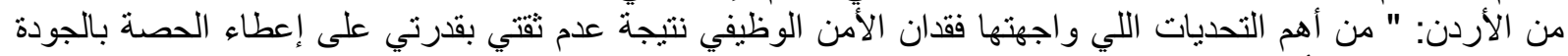

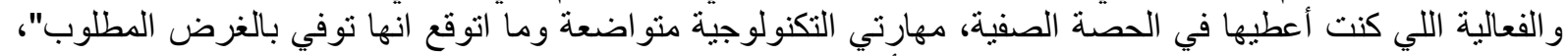

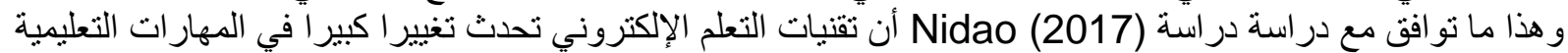

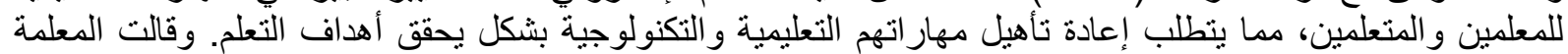

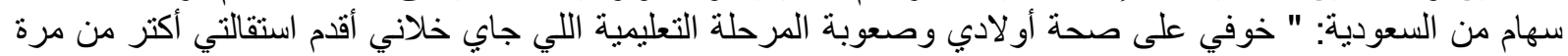

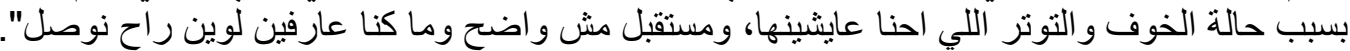

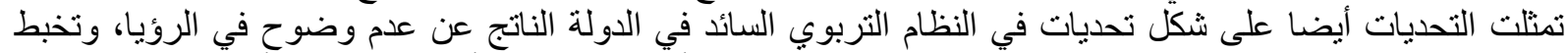

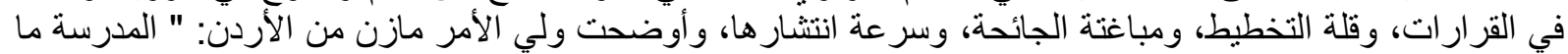

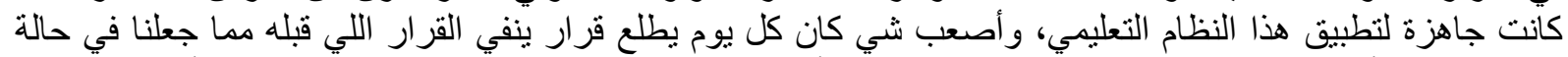

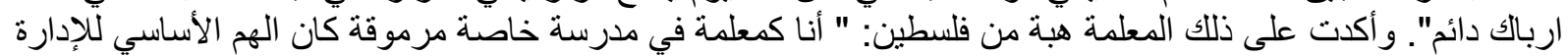

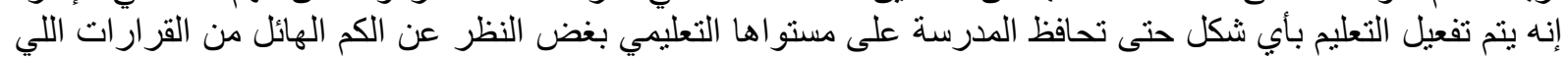

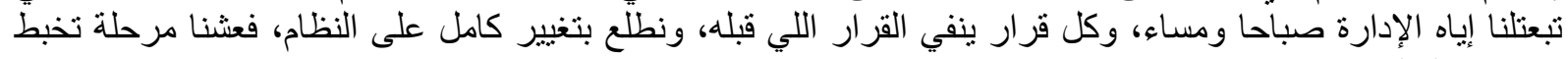

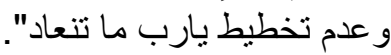
وبالرغم من كل التحديات و والصعوبات التي واجيات واجتت توظيف الاستر اتيجيات التعلمية في التعليم الإلكتروني إلا أن المعلمون

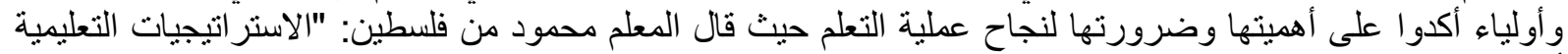

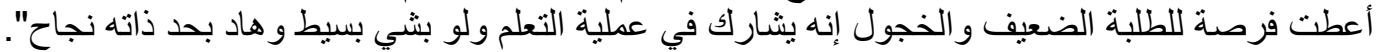

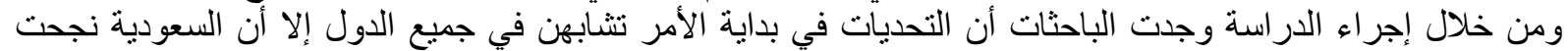

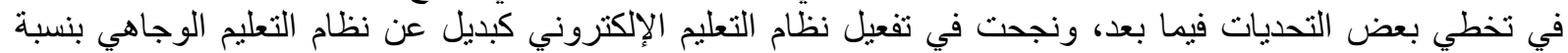

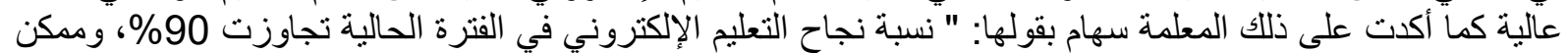

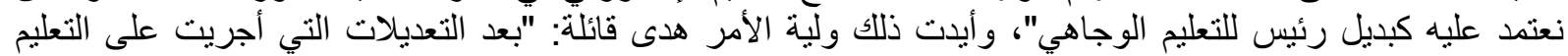

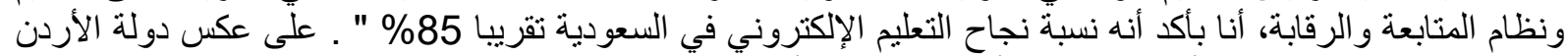

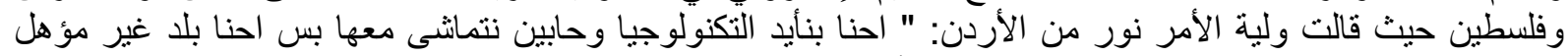

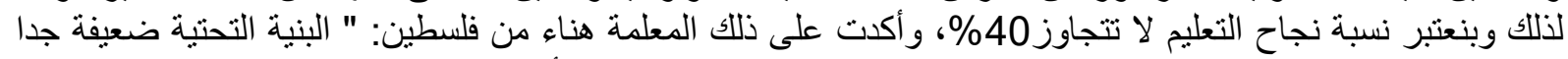

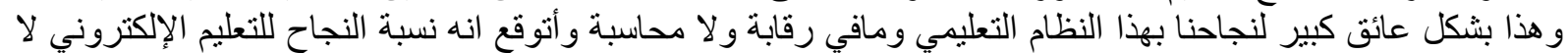

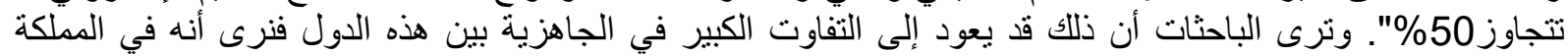

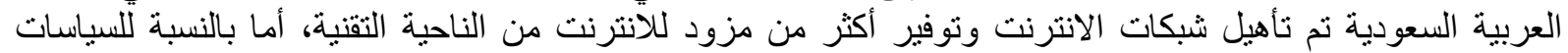

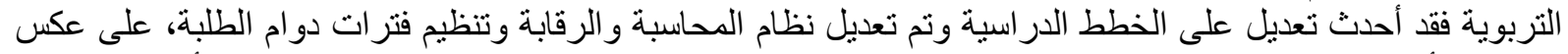

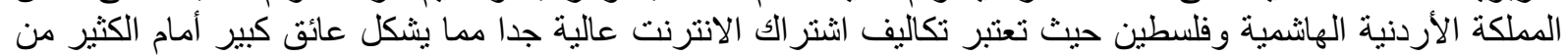

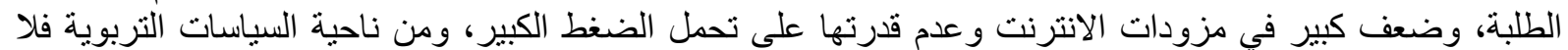

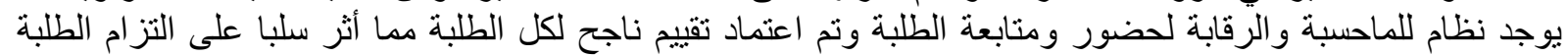
بهذا النظام التعليمي و التفاعل معه.

ثالثا: مقترح لتطوير توظيف الاستراتيجيات التعلمية في التعلم الإكتروني في ظل الأزمات. 


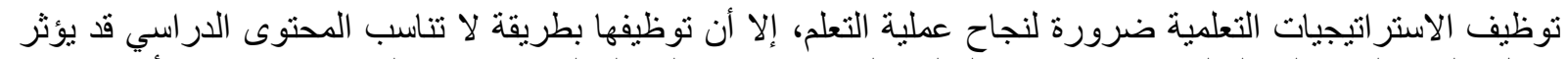

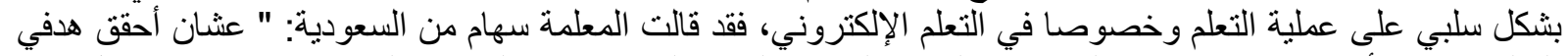

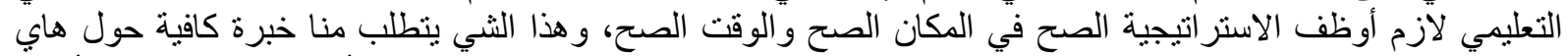

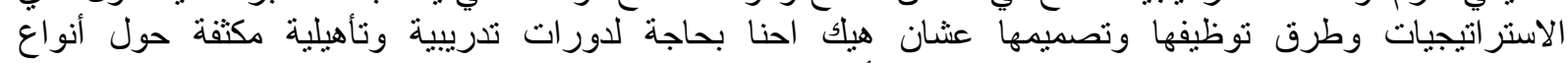

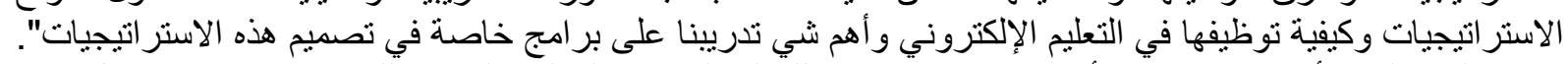

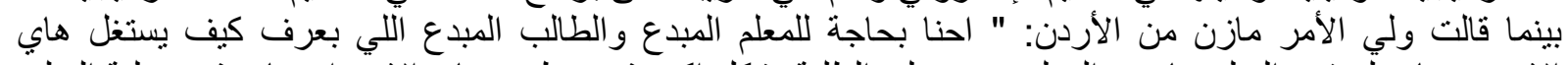

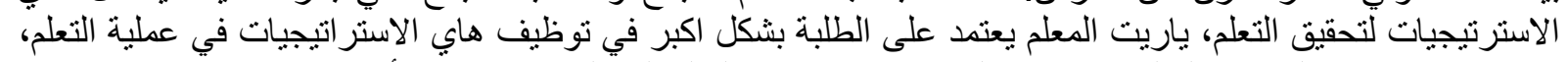

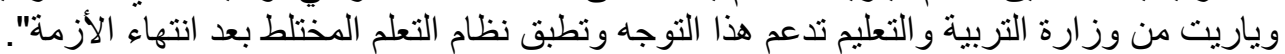

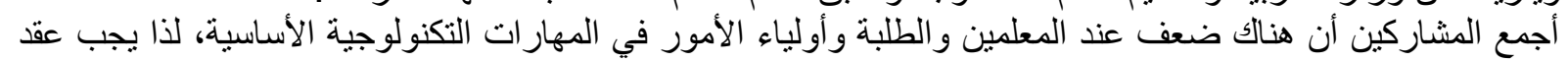

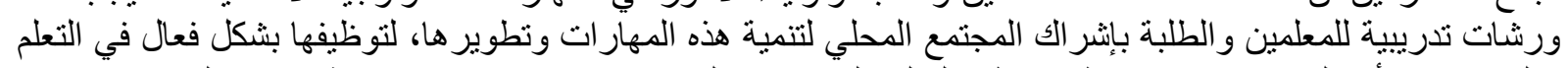

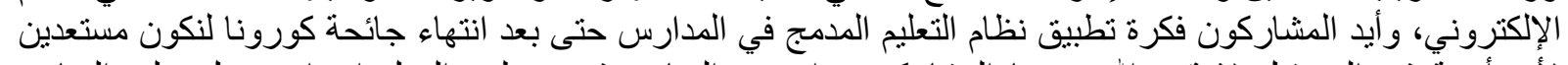

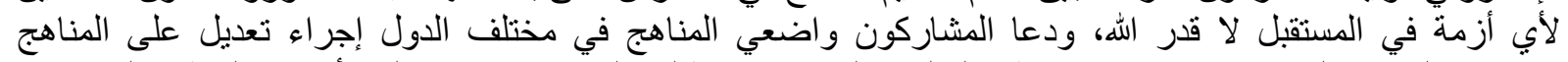

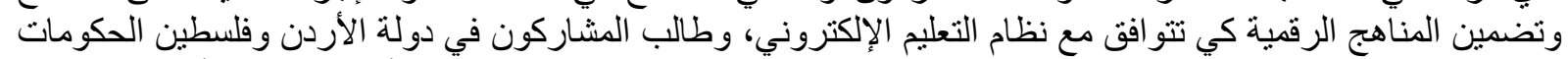

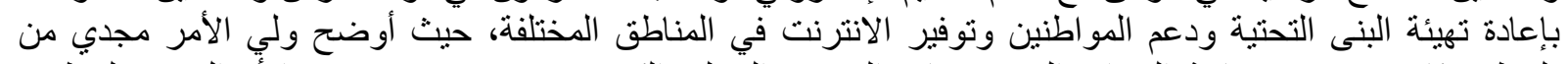

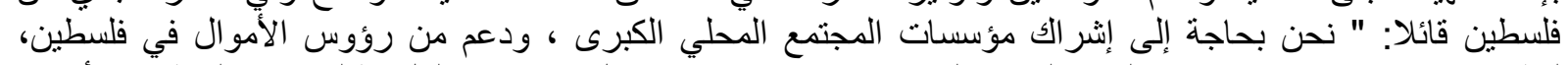

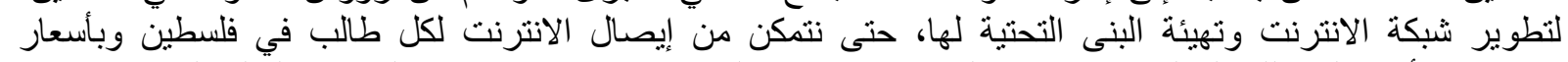

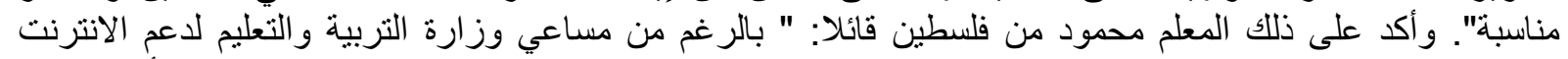

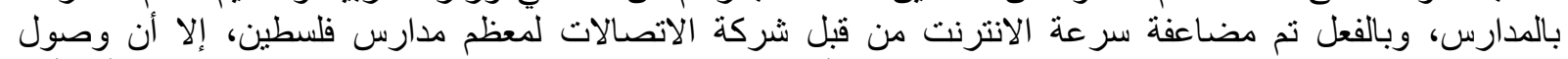

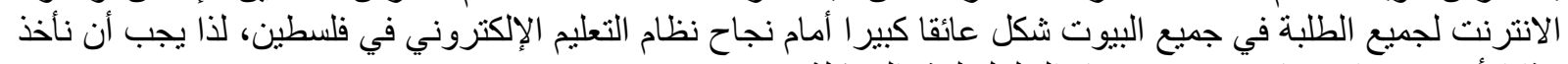

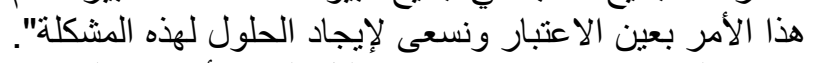

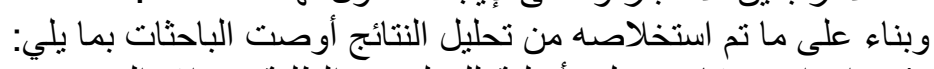

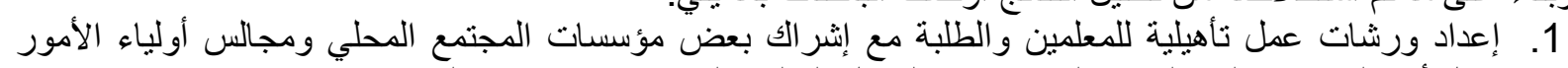

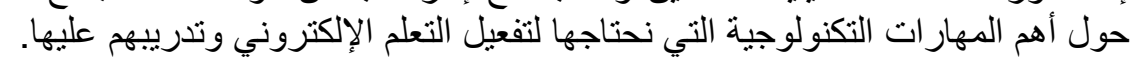

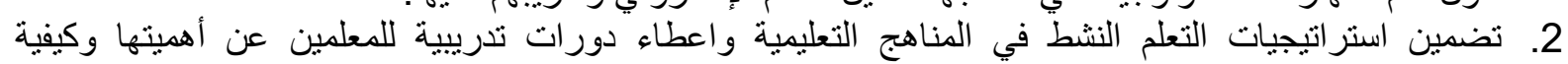

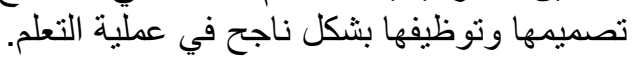

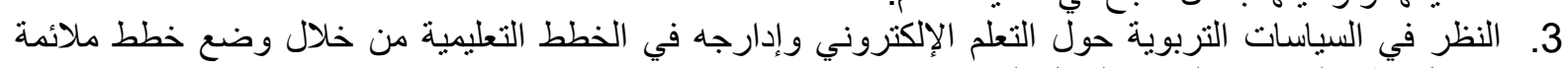

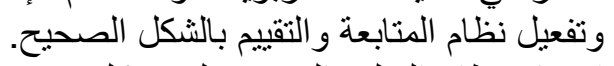

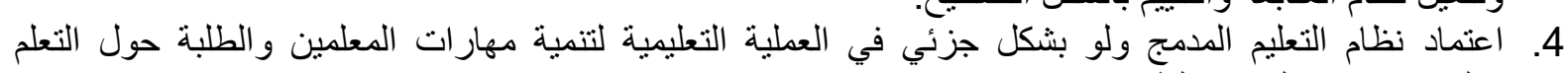

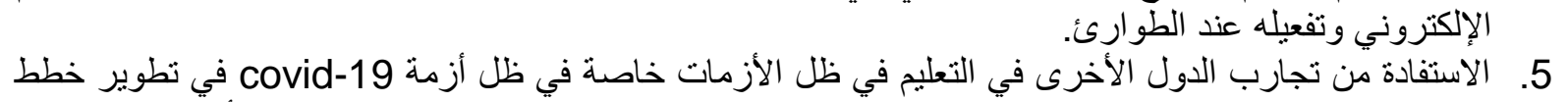

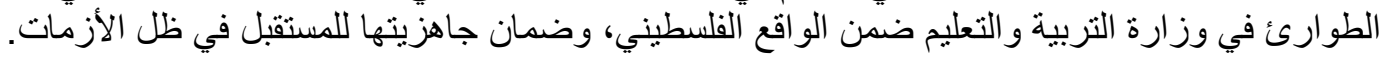

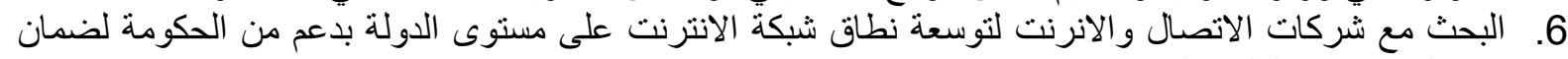
وصول الانترنت لكل طالب.

العرسان، سامر (2017). فاعلية استخدام استر اتيجيات التعلم النشط المستندة إلى النظرية المعرفية الاجتماعية في تتمبة

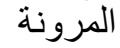
المعرفية ودافعية الإنجاز الأكاديمي لدى طلاب قسم علم النفس بجامعة حائل، مجلة القدس المفتوحة للأبحاث والدراسات

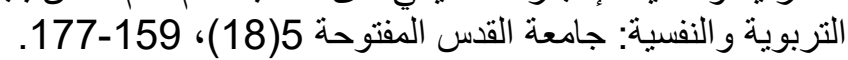

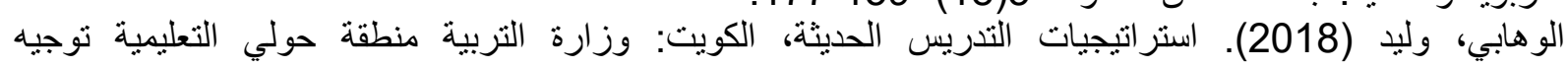

References:

Aboagye, E., Yawson, J. A., \& Appiah, K. N. (2020). COVID-19 and E-learning: The challenges of students in tertiary institutions. Social Education Research, 1-8. 
Alersan, S. (2017). The effectiveness of using active learning strategies based on social cognitive theory in developing resilience Cognitive and academic achievement motivation among students of the Department of Psychology at the University of Hail. (in Arabic). Al-Quds Open Journal for Research and Studies Educational and psychological, 5(18), 159-177.

Alfaqeeh, A. (2017). Designing Qualitative Research in the Educational Field with a Focus on Research on Teaching Arabic Language. International Journal of Educational Psychological Studies, 441(5945), 1-15.

Alhabeeb, A., \& Rowley, J. (2017). Critical success factors for eLearning in Saudi Arabian universities. International Journal of Educational Management, 31(2), 131-147. https://doi.org/10.1108/IJEM-01-2016-0006

Alwahabi, W. (2018). Modern Teaching Strategies. (in Arabic). Kuwait: Ministry of Education, Hawally Educational District, Social Guidance.

Anwer, F. (2019). Activity-Based Teaching, Student Motivation and Academic Achievement. Journal of Education and Educational Development, 6(1), 154-170.

Ary, D., Jacobs, L. C., \& Razavieh, A. (2010). Introduction to research in education 8th edition, Wardswoth Cengage Learning. Canada: Nelson Education Ltd Exotic Classic.

Barak, M., Watted, A., \& Haick, H. (2016). Motivation to learn in massive open online courses: Examining aspects of language and social engagement. Computers and Education, 94, 49-60. https://doi.org/10.1016/j.compedu.2015.11.010

Castillo, M. C. Del Á., \& Córdova, K. E. G. (2014). Language learning strategies and academic success: A Mexican perspective. Universitas Psychologica, 13(2), 703-713. https://doi.org/10.11144/Javeriana.UPSY13-2.Ilsa

Chen, M. L. (2014). Age differences in the use of language learning strategies. English Language Teaching, 7(2), 144-151. https://doi.org/10.5539/elt.v7n2p144.

Dornyei, Z., \& Ryan, S. (2015). The psychology of the language learner revisited. Routledge.

Essam, S., \& Al-Ammary, J. (2013). The impact of motivation and social interaction on the e-learning at Arab Open University, Kingdom of Bahrain. Creative Education, 4(10), 21.

Griffiths, C. (2018). The strategy factor in successful language learning: The tornado effect. Multilingual Matters

Gros, B., \& García-Peñalvo, F. J. (2016). Future trends in the design strategies and technological affordances of e-learning. Springer

Gursoy, E. (2010). Investigating Language Learning Strategies of EFL Children for the Development of a Taxonomy. English Language Teaching, 3(3), 164175. https://doi.org/10.5539/elt.v3n3p164

Fandiño, F. G., Muñoz, L. D., \& Silva Velandia, A. J. (2019). Motivation and ELearning English as a foreign language: A qualitative study. Heliyon, 5(9). https://doi.org/10.1016/j.heliyon.2019.e02394

Fırat, M., Kılınç, H., \& Yüzer, T. V. (2018). Level of intrinsic motivation of distance education students in e-learning environments. Journal of Computer Assisted Learning, 34(1), 63-70. https://doi.org/10.1111/jcal.12214 
Fryer, L. K., \& Bovee, H. N. (2016). Supporting students' motivation for e-learning: Teachers matter on and offline. Internet and Higher Education, 30, 21-29. https://doi.org/10.1016/j.iheduc.2016.03.003

Hikmawan, T., Sutarni, N., \& Hufad, A. (2019). The role of electronic learning media in creativity learning. Journal of Physics: Conference Series, 1375(1). https://doi.org/10.1088/1742-6596/1375/1/012030

Ismaeel, D. A., \& Al Mulhim, E. N. (2019). Impact of e-learning strategies to design eportfolio on achievement motivation and product quality. International Journal of Distance Education Technologies, 17(2), 59-73. https://doi.org/10.4018/IJDET.2019040104

Kormos, J., \& Csizér, K. (2014). The Interaction of Motivation, Self-Regulatory Strategies, and Autonomous Learning Behavior in Different Learner Groups. TESOL Quarterly, 48(2), 275-299. https://doi.org/10.1002/tesq.129

Levings, J. M. (2015). A multiple case study of professional development and perspective change within the cooperative extensive service. Dissertation Abstracts International Section A: Humanities and Social Sciences, 76(2-A (E)), No-Specified. http://gateway.proquest.com/openurl?url_ver=Z39.88-

Lin, M. H., Chen, H. C., \& Liu, K. S. (2017). A study of the effects of digital learning on learning motivation and learning outcome. Eurasia Journal of Mathematics, Science and Technology Education, 13(7), 3553-3564. https://doi.org/10.12973/eurasia.2017.00744a

Linton, J. N. (2017). Institutional factors for supporting electronic learning communities. Online Learning Journal, 21(1), 238-256. https://doi.org/10.24059/olj.v21i1.953

Mazumder, Q. H. (2014). Student Motivation and Learning Strategies of Students from USA, China and Bangladesh. International Journal of Evaluation and Research in Education (IJERE), 3(4). https://doi.org/10.11591/ijere.v3i4.6288

Moubayed, A., Injadat, M., Nassif, A. B., Lutfiyya, H., \& Shami, A. (2018). E-learning: Challenges and research opportunities using machine learning \& Data analytics. IEEE Access, 6, 39117-39138.

Murphy, M. P. A. (2020). COVID-19 and emergency eLearning: Consequences of the securitization of higher education for post-pandemic pedagogy. Contemporary Security Policy, 41(3), 492-505. https://doi.org/10.1080/13523260.2020.1761749

Naidoo, V. (2017). Challenges Facing E-Learning. In Multiculturalism and Technology-Enhanced Language Learning (pp. 271-288). IGI Global.

University, G. (2020). Relationship between High School Students' Motivation Levels and Learning Strategies. International Journal of Progressive Education, 16(3), 0-2. https://doi.org/10.29329/ijpe.2020.248.5

Shi, H. (2017). Learning strategies and classification in education. Institute for Learning Styles Journal, 1(1989), 24-36.

Suwanarak, K. (2019). Use of learning strategies and their effects on English language learning of thai adult learners. 3L: Language, Linguistics, Literature, 25(4), 99-120. https://doi.org/10.17576/3L-2019-2504-07 
Vannoni, M. (2015). What Are Case Studies Good for? Nesting Comparative Case Study Research into the Lakatosian Research Program. Cross-Cultural Research, 49(4), 331-357. https://doi.org/10.1177/1069397114555844

Vibulphol, J. (2016). Students' Motivation and Learning and Teachers' Motivational Strategies in English Classrooms in Thailand. English Language Teaching, 9(4), 64. https://doi.org/10.5539/elt.v9n4p64

Wael, A., Asnur, M. N. A., \& Ibrahim, I. (2018). Exploring Students' Learning Strategies in Speaking Performance. International Journal of Language Education, 2(1), 65. https://doi.org/10.26858/ijole.v2i1.5238

Yildirim, I., Cirak-Kurt, S., \& Sen, S. (2019). The effect of teaching "learning strategies" on academic achievement: A meta-analysis study. Eurasian Journal of Educational Research, 2019(79), 87-114. https://doi.org/10.14689/ejer.2019.79.5 